\title{
Social Marketing, Shock Advertising, and Risky Consumption Behavior
}

\begin{abstract}
Purpose: The overall objective of this research is to "explore whether shock advertising has a long-lasting positive effect on the smoking cessation among smoking Jordanians in a country where smoking is a deep-rooted social norm."

Design/methodology/approach: This research is an exploratory qualitative research. A purposeful sampling technique was used to select participants from a mall intercept and randomly divided into groups of seven. Each group was interviewed in two different focus group sessions (four weeks apart). All focus groups were audio-recorded, transcribed, and analyzed using thematic analysis.

Findings: A total of 41 participants took part in the focus group session. Most participants were smokers or second-hand smokers. "Three overarching themes were generated from the focus groups: previous anti-smoking campaign experience, shock advertising (SA) impact, and drawbacks of SA. All participants reported that they have never been exposed to shocking adverts, and the shock appeal has never been applied in any of the anti-smoking or health awareness campaigns in Jordan. This research revealed that incorporating images of children with a mixture of emotional and fear appeals is effective in targeting Jordanian parents' negative consumptive behaviors, which may harm other individuals, especially children. Moreover, most participants commented that the effects of shock adverts would be very short term and would not likely change behaviors".
\end{abstract}

Originality/Value: This research contributes both 'theoretically and practically to the value and effectiveness of shock advertising. This research area is overlooked in MENA countries, particularly Jordan”.

Keywords: Shock advertising, Social marketing, Smoking, Image Elicitation, MENA. 


\section{Social Marketing, Shock Advertising, and Risky Consumption Behavior}

\section{Introduction}

Smoking is a global epidemic that draws a lot of attention, as it poses threats to health and society. Arab countries are steadily becoming the leading tobacco users worldwide, and with the prevalence of waterpipe smoking as well, there has been an increase in the number of smokers (Darawad et al. 2020). In the "Arab world, smoking is more of a social problem. Smoking has health effects that reach nonsmokers as well. In 2019, it was estimated that 7 million deaths worldwide, primarily of women and children, were caused by second-hand smoke (SHS) inhalation. It also causes more deaths than alcohol, suicide, HIV/AIDS, homicide, and drugs taken together worldwide. Furthermore, it has a negative effect on the well-being of infants, children and other nonsmokers (World Health Organization 2020). Smoking is the cause of 8 million deaths annually, and it is more popular in low- and middle-income countries than in high-income countries" (Feigl et al. 2015).

The problem of smoking "extends beyond the smoking consumers themselves. In 2011, it was estimated that 600,000 deaths were caused by SHS worldwide. Most of those deaths were women and children (Kusel, Timm \& Lockhart 2013). Exposure to SHS has harmful consequences to children's health, it increases the risk of acquiring infections (e.g., otitis media), deterioration of lung functions, invasive meningococcal infections, and asthma exacerbations" (Huque et al. 2015).

\section{Smoking and its cultural implications}

Smokers usually start the "habit at a young age mainly under friends' influence, it contributes to building friendships and many other forms of relationships as it brings people from different backgrounds" (Albert-Lörincz et al. 2019). Moreover, "smoking became a tool for escapism and a way to cope with stress and anxiety, as opposed to labelling smoking as a deviant habit (Gooch, Sheldon 2019). Due to the alarming results of studies revealing the risks of cigarette smoking, many smokers in Jordan have shifted to waterpipe smoking. In the Arab world, waterpipe smoking has become a serious health risk given the widespread of this habit among adolescents and young adults (Hammal et al. 2008). It has been reported that waterpipe smoking has expanded the social aspect of smoking as it is often practiced among a group of friends with elaborate rites for the preparation of the tools or apparatus for smoking. The waterpipe itself is the center of a social 
activity and fuel for conversation while one is leisurely enjoying the company of friends" (Knishkowy, Amitai 2005).

Arab countries are steadily becoming the leading tobacco users worldwide and with the prevalence of waterpipe smoking, there has been an increase in the number of smokers. In Jordan, "smoking rates are of the highest in the world. More than eight out of 10 Jordanian men consume nicotine products (e.g., cigarettes, e-cigarettes, waterpipe) (Safi, Al-Tahat 2020). A recent research by the World Health Organization (WHO) reported that tobacco smoking is prevalent among 66\% and $17 \%$ of Jordanian men and women, respectively (Safi, Al-Tahat 2020, Burki 2019). Experts believe that the smoking rate among Jordanian women could be underreported since women smoking cigarettes have historically been stigmatized in Jordan (Burki 2019). Moreover, the researcher reported that $62 \%$ of young adults are regularly exposed to SHS. Evidence from Jordan suggests high tobacco consumption by health workers ( $46.3 \%$ of physicians and $41.5 \%$ of nurses) (Shishani et al. 2011). Unfortunately, the consumption of nicotine products is not exclusively practiced by Jordanian adults. Nearly one-third of schoolchildren in Jordan (37.2\% of schoolboys and $23.7 \%$ of schoolgirls) initiated cigarette smoking by the $10^{\text {th }}$ grade (Haddad et al. 2011). Smoking and SHS problem is also prevalent in other Arabic countries" (Sibai et al. 2016).

Smoking cigarettes in Jordan is not the only problem. Smoking waterpipes (shisha, hookah, or narghile) is equally popular and is considered a social norm, especially among teenagers. As many as $61.1 \%$ of university students smoked or still smoke waterpipes, while in some communities, parents encourage their children to smoke these too. "Smoking waterpipes is fashionable among young women as well. Waterpipe coffee shops strategically located every 200 to 300 meters in Jordanian cities (Haddad et al. 2011) complicate matters. Moreover, since there is a strong tie between smoking and culture, the desire to smoke less while also socializing in a smokingencouraging environment presents real challenges for social marketers in Jordan. The growing popularity of waterpipe smoking has created a new epidemic in Arab countries. The prevalence of waterpipe and e-cigarettes smoking can also be associated with the common fallacy that it is less risky than cigarette smoking (Karasneh et al. 2021). Though, research has concluded that waterpipe smoking poses the same health risk as smoking tobacco" (Kamal 2017). 


\section{Social marketing and shock advertising}

Social marketing is defined as the "adaptation and adoption of commercial marketing activities, institutions, and processes as a means to induce behavioral change in a targeted audience on a temporary or permanent basis to achieve a social goal" (Dann 2010). Social marketers need to deeply understand behavioral change barriers (e.g., addiction) to achieve an effective and positive behavioral change for unwholesome behaviors. This strategy "aids the target audience to perceive the benefit of changing the unwholesome behavior (Lee, Kotler 2011). Moreover, social marketers need to elicit opinions of various target groups (e.g., young adults, minorities, ethnic groups) to launch an effective social marketing campaign" (Keller et al. 2014).

Anti-smoking campaigns regularly rely on "emotional adverts to highlight illnesses caused by smoking. This type of campaign aims to influence emotion-based processing of risk information and bring attention to messages delivered in competitive media environments to ultimately create behavioral change (Albouy, Décaudin 2018). Moreover, anti-smoking campaigns employ graphic images on cigarette packages as they are more effective than text information (Nan et al. 2015) to build strong, emotionally driven adverts to encourage the target audience to quit smoking amongst" (Farrelly et al. 2012).

Adverts and communications' effectiveness are often measured by recipients' ability to recall the commercial content such as the brand name and the advertised message (Jin, Kerr \& Suh 2019). Therefore, "memorable campaigns resonate with the audience and build a connection between the customer and product (Machová, Huszárik \& Tóth 2016). Therefore, this current research employed different types of adverts to evaluate the effectiveness of social marketing campaigns that employ imagery as a SA technique to counter-market unwholesome behaviors".

Shock Advertising is a "powerful advertising tactic to capture the target audience's attention and encourage advertising processing, as it deliberately violates social norms" (Dahl, Frankenberger \& Manchanda 2003). Grabbing consumer attention is very challenging and essential for marketers, as consumers are bombarded by thousands of adverts daily (Wright et al. 2016). Recently, "the advertising environment is highly competitive, this intense competition drove marketers to search for more effectual strategies grabbing the audience attention. Consequently, shock or controversial advertising became an accepted advertising strategy for both profit and not-for-profit institutes (Parry et al. 2013). The nineties of the last century witnessed the elevation of SA communicating political and social messages with the audiences, promoting international harmony and drawing the attention to major societal problems". 
On the other hand, emotional appeals are concerned with consumers' social and psychological needs that drive their wants and purchase decision. Emotional appeal "stands for the effective text that will activate the emotions of the audience to accept an idea, proposition or perform an action" (Jabbar, Christopher 2012). Emotions evoked in advertising can "either be positive (e.g., love and contentment) or negative (e.g., fear, disgust, anger, and sadness)" (Taute, McQuitty \& Sautter 2011). Emotions are "more effective than listing information and facts to influence consumers' decisions regarding product brands. Therefore, emotional advert appeals increase the likelihood that consumers make a purchase or change a behavior. Similarly, shocking imagery can be used to evoke fear in audiences as an emotional response to an advert (Hastings 2007). Similar negative emotions (e.g., fear or threat) have been employed to encourage attitude and behavior changes in different areas that include smoking cessation (Simpson 2017). Evidence suggests that campaigns that employ only emotional content performed about twice as effective as campaigns with purely rational content (Brainfluence 2012). This is a rational finding, considering that people are expected to feel first and think second".

Shock appeal and advertising are effective in targeting social problems. Evidence suggests that "shock appeal results in better memory recall when compared to other appeals (i.e., fear and information appeals) (Dahl, Frankenberger \& Manchanda 2003). SA outcomes are not limited to grabbing attention, but it is also effective at creating recall and inducing behavioral change (Dahl, Frankenberger \& Manchanda 2003). Some audience groups, however, can be more responsive to emotional adverts. Elderlies are found to be more responsive to shock adverts than youth (Albouy, Décaudin 2018) and other researchers reported that responsiveness to shock adverts is correlated with gender, ethnicity and background culture (Engelbart, Jackson \& Smith 2017). However, SA does not guarantee changing tentative buying decisions (Prendergast, Ho \& Phau 2002), or successfully establishing the intended behavioral change (Krstic 2007). There is no clear evidence to conclude that SA can have an actual impact on the actual behavioral change of the target audience, and this requires further research to explore this area of research".

\section{Shock advertising and smoking}

The effectiveness of SA in targeting smoking is questionable. Though some studies support "the effectiveness of SA in smoking cessation campaigns, other studies highlight its failure, mainly due to the addictive nature of smoking. Furthermore, graphic warnings featuring graphic depictions of diseases were significantly efficient among Mexican youth and adults (Hammond et al., 2012). Furthermore, twenty percent of participants to a research assessing the efficacy of SA applied in 
the Get Unhooked campaign in the UK reported willingness to quit (Veer et al., 2008). The Truth campaign in the USA targeted American youth of ages 12-17 with smoking prevention content found that social marketing anti-smoking campaigns targeting hipsters in the USA were effective" (Ling et al., 2014).

On the other hand, Hastings (2007) expressed doubt about the effectiveness of anti-smoking adverts for regular smokers and people who usually live in an environment where smoking is a norm (Cited in Corner and Randal, 2011). According to MacFadyen, Stead \& Hastings (1999) "smokers face anti-smoking campaigns with denial and they put themselves in a defensive state, mostly accompanied by emotions of anxiety and guilt as a result of the addictive nature of the product (very high involvement level) as smokers are struggling to quit smoking (MacFadyen, Stead \& Hastings 1999). Schiffman and Wisenblit mentioned that smokers or any person with an unhealthy habit usually deny potential personal disaster, such as that illness or problems will happen to him/her" (Schiffman, Wisenblit 2019). Therefore, the choice to quit smoking is a very high involvement decision that a consumer makes, and smokers would need a comprehensive campaign including various tools that include SA to succeed in quitting.

Though some existing research assesses "the effect of shock advertising on smoking and smokers' behaviors, most of the previous research was conducted in developed countries and did not focus directly on smoking nor targeted SHS. Therefore, the overall objective of this research is to explore whether shock advertising has a long-lasting positive effect on the smoking cessation among smoking Jordanians in a country that smoking is a deep-rooted social norm". The Precise objectives of this research are to explore: 1) "what emotions evoked amongst participants after exposure to shock adverts targeting smoking and SHS, 2) how shock adverts affect whether smokers expose others to SHS, and 3) what supporting and challenging factors impact the effectiveness of shock advert in inducing behavioral change".

\section{Methodology}

This research is an exploratory qualitative research that aimed to "explore whether shock advertising has a lasting positive impact on the smoking cessation in Jordan. Therefore, this research with exploratory nature commonly uses qualitative methods as they intend to achieve a depth of understanding, interpretation, and contextualization of data (Cleland 2015). Unlike quantitative methods, qualitative methods are inductive and can generate hypotheses and theories 
that relate to participants' perception, attitudes, emotions, and beliefs following the exposure to shock adverts targeting smoking” (Charmaz, Thornberg 2020, Corbin, Strauss 1990).

\section{Anti-smoking advert selection}

Prior to the pilot stage, "one researcher conducted a Google image search for relevant shock adverts. Adverts with the highest relevance on Google search engine were initially chosen at the time of the research design $(n=25)$. Following the Google image search, a panel of experts in marketing and advertising reviewed the 25 adverts, and were shortlisted to ten adverts. The panel of experts made sure that the shortlisted adverts covered the shock appeals suggested by Dahl et al. (2003) and took into consideration the conservative and religious nature of the Jordanian society".

\section{Pilot stage}

For the pilot stage, "a group of collogues and graduate students were invited to take part in a pilot stage. The purpose of this pilot stage was to assess and refine the focus interview guide and questions. The pilot stage also aimed to increase confidence and training for the researchers conducting the focus groups. Moreover, the pilot stage aimed to finalize the list of images and adverts that will be used for the main data collection".

A total of eight participants took part in the pilot research (five males and three females). "Six of the participants in the pilot research were smokers and the remaining two were nonsmokers. The first focus group lasted for 106 minutes, and a second focus group was conducted four weeks later and lasted for 67 minutes. Participants provided comments to improve the clarity and wording of some questions, and six of them recommended to take out one of the adverts. The focus group questions were refined according to the pilot group comments, and nine adverts were used for the main data collection" (Table 1). The results from the pilot research were not included in the results of this research. 
Table 1: A summary and descriptions of advertisements used

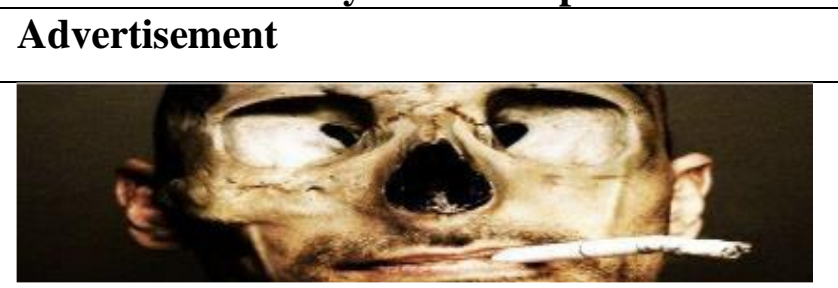

\section{Description}

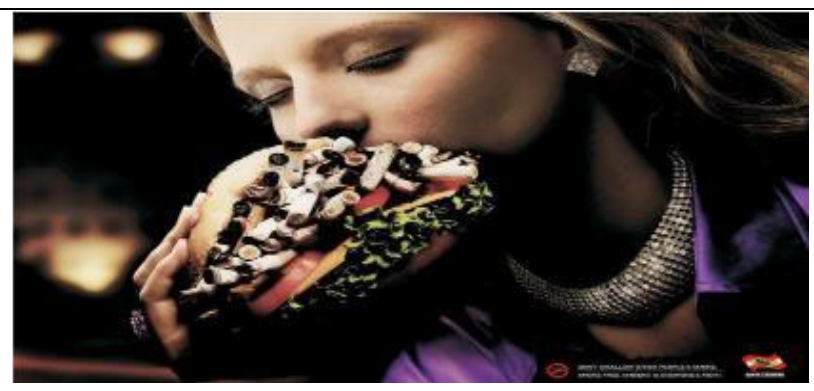

"An advertisement from Brazil showing a woman eating a burger full of cigarettes quoting" "Don't swallow other people's smoke."

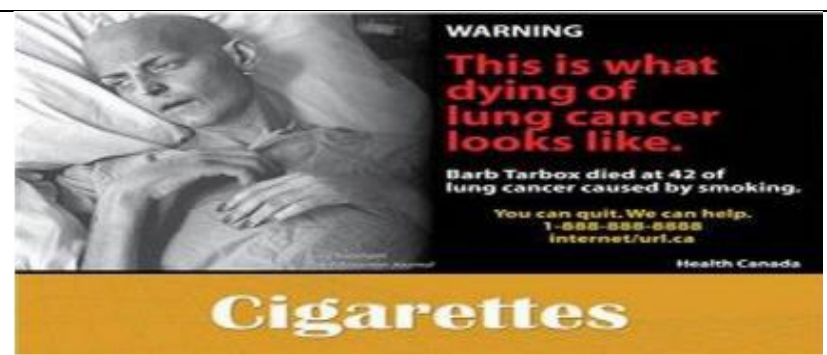

"A Canadian advertisement showing a dying mother quoting" "This is what dying of lung cancer looks like."

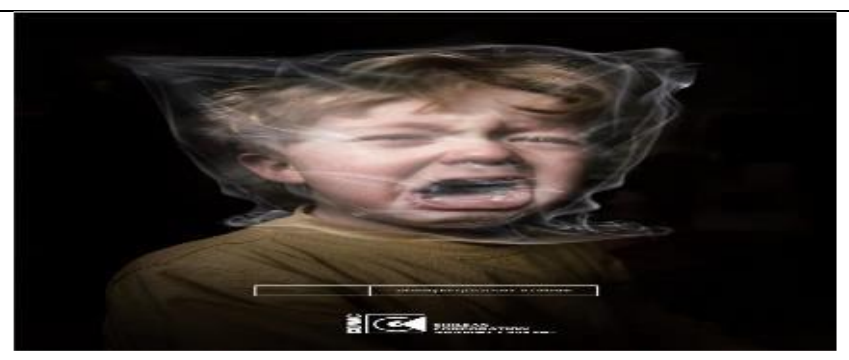

"An advertisement from Chile representing a child suffocating in a bag wrapped around his head."

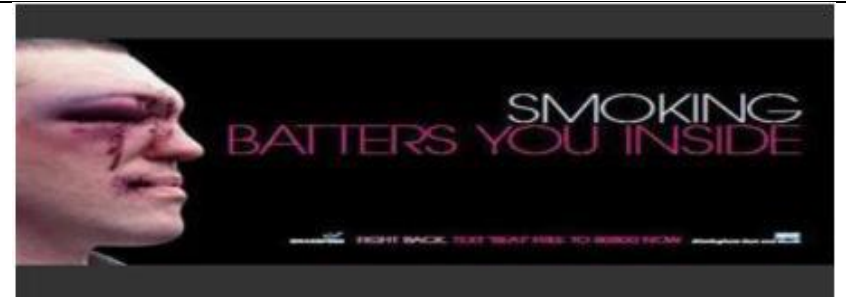

"A UK-based advertisement encouraging smokers to quit by representing a fighter with bleeding eyes."

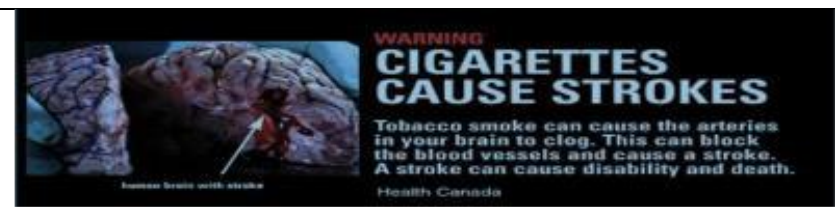

"An advertisement from Canada showing how a stroke looks like within the brain."

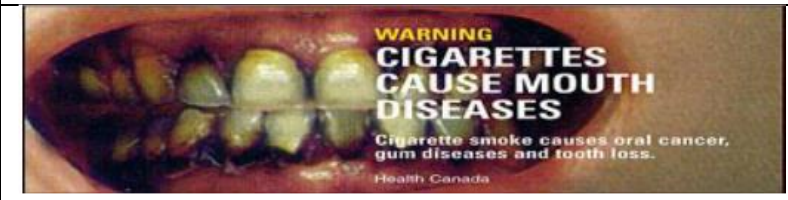

"A Canadian image showing the mouth and teeth of a smoker." 


\begin{tabular}{|l|l|}
\hline "A UK Based advertisement showing a \\
woman hooked from the mouth quoting" \\
"get unhooked"
\end{tabular} \mid $\begin{aligned} & \text { "A UK based advertisement showing a } \\
& \text { cigarette stuffed in fatty tissue quoting" } \\
& \text { "Every Cigarette we smoke makes fatty } \\
& \text { deposits in our arteries." }\end{aligned}$

\section{Data collection}

\section{Participants}

Participants were invited to take part in this research through a mall intercept approach. "Mall intercept is one of the most used data collection approaches in advertising and marketing research. Mall intercepts provide a relatively quick and economical way for data collection, especially of hard-to-reach segments of a population” (Rice, Hancock 2005).

In this research, "shoppers at a mall located in a large metropolitan city in Jordan were solicited and invited by a trained researcher. The researchers gave these shoppers a brief description about the research objectives and protocol and invited them to fill a data collection form. This data collection aimed to collect potential participants' sociodemographic information (e.g., age, gender, educational levels, smoking habits), and their willingness and availability to participate in two focus group sessions. A purposeful sampling technique was used to select 42 participants from the mall intercept respondents, and participants were randomly divided into groups of seven. One participant withdrew from the research prior to defining the first focus group. The researchers agreed that data and theoretical saturation had been reached following the sixth focus group when redundancy in new data and no new additional information were being found" (Charmaz, Thornberg 2020).

\section{Focus groups}

In this research, "41 participants were randomly divided into six focus groups. All participants were asked to provide consent for their anonymized responses to be published. Each group was interviewed in two different sessions (four weeks apart). The focus interview guide is available in 
appendix 1. All focus groups were audio-recorded, and all recordings were transcribed and checked for accuracy".

In the first focus group session, "participants were exposed to a set of adverts (Table 1) and were asked to discuss their opinions about this type of marketing communication strategies. Participants were also asked to describe how they perceived shock adverts, how they felt toward them, whether the adverts were ethically justified and how these adverts would influence their behaviors and attitudes toward smoking. The first focus group session ran for an average of 102 minutes across all the six groups (Max: 116 minutes, Min: 95 minutes)”.

The second session of the focus groups aimed to "assess participants' memory recall and explore emotions that were developed after the first group's focus session. The researchers asked participants what they remembered about the adverts from the first session and how they felt about them in the last four weeks. Participants were also asked to express their opinions regarding the usefulness of shock advertising as a marketing communication strategy and in creating social and health awareness. Moreover, participants were asked about their opinions regarding effective marketing strategies that can be used to create social and health awareness in Jordan. The second focus group session ran for an average of 61 minutes across all the six groups" (Max: 67 minutes, Min: 55 minutes).

\section{Data analysis}

To address our research questions, "transcripts were analyzed using thematic analysis. This method is useful for identifying and analyzing patterns of meaning in qualitative research (Clarke, Braun 2014). Unlike other methods used in qualitative analysis, thematic analysis is not tied to a particular epistemological or theoretical perspective" (Maguire, Delahunt 2017).

Two researchers (I.L.M and T.L.M) read and re-read the transcripts to get a sense of data and become familiar with it. The two researchers identified initial codes (i.e., themes) that were relevant to the research questions, and independently coded transcripts and searched for new themes. All themes and subthemes identified were reviewed, defined, discussed, and agreed on by the three researchers (I.L.M, T.L.M, and M.B.N) until a consensus was reached. In order to increase trustworthiness of findings, member check and analyst triangulation (multiple researchers reviewed findings) were employed to decrease threats to credibility. Moreover, the researchers decreased threats to conformability using audit trails describing all research steps taken (Lincoln, Guba 1985). 


\section{Findings}

A total of "41 participants took part in the focus group session. Most participants were smokers or second-hand smokers (Table 2). Nearly half of the participants were males $(n=22)$, and the majority had a university degree. Participants' age range was 18-35 years, and nearly $20 \%$ of them were married with children" (Table 2).

Table 2: Sociodemographic characteristics of participants $(n=41)$

\begin{tabular}{|l|l|l|}
\hline Characteristics & $\mathbf{n}(\mathbf{\%})$ \\
\hline \multirow{4}{*}{ Gender } & Male & $22(53.6 \%)$ \\
\cline { 2 - 3 } & Female & $19(46.3 \%)$ \\
\hline \multirow{5}{*}{ Mevel } & Up to secondary education & $12(29.2 \%)$ \\
\cline { 2 - 3 } & $\begin{array}{l}\text { Undergraduate (diploma and } \\
\text { bachelor's degree) }\end{array}$ & $20(48.8 \%)$ \\
\cline { 2 - 3 } & Postgraduate degree & $9(22 \%)$ \\
\hline \multirow{3}{*}{ Smoking status } & Single & $31(75.6 \%)$ \\
\cline { 2 - 3 } & Married with Children & $8(19.5 \%)$ \\
\cline { 2 - 3 } & Married without Children & $31(7.9 \%)$ \\
\cline { 2 - 3 } & Non-smokers & $10(24.4 \%)$ \\
\hline Age range & & $18-35$ years \\
\hline
\end{tabular}

Three overarching themes were identified in the focus groups: "previous anti-smoking campaign experience, shock advertising impact, and drawbacks to SA. Overarching themes and sub themes emerging from this research are present in Table 3".

Table 3: Overarching themes and subthemes

\begin{tabular}{|c|c|}
\hline Overarching Themes & Sub themes \\
\hline \multirow{2}{*}{$\begin{array}{l}\text { 1. "Previous anti-smoking campaign } \\
\text { experience." }\end{array}$} & "First shock advertising experience." \\
\hline & $\begin{array}{l}\text { "Current Anti-smoking Campaigns are } \\
\text { Ineffective." }\end{array}$ \\
\hline \multirow[t]{3}{*}{ 2. "Shock Advertising Impact." } & "Triggering Emotions." \\
\hline & "Memory Recall." \\
\hline & “Concerns for SHS.” \\
\hline \multirow[t]{3}{*}{ 3. "Drawbacks to shock advertising." } & "Accustomed to Shocking Images." \\
\hline & "Defensiveness triggered by Offensiveness." \\
\hline & "Capturing but Ineffective." \\
\hline
\end{tabular}




\section{Previous anti-smoking campaign experience}

Two subthemes were identified under this overarching theme: First "Shock Advertising Experience and Current Anti-smoking Campaigns Are Ineffective. All participants reported that they have never been exposed to shocking adverts in Jordan. The shock appeal has never been applied in any of the anti-smoking or health awareness campaigns the participants were aware of or recalled".

The last results provided significance of this research, "as the research sample lives in a low- to middle-income country, where smoking is rooted in the culture and day by day behavior, and antismoking regulations are neglected and ineffective (Ekpu, Brown 2015), and has never been exposed to any type of SA targeting smoking, will view such images for the first time. The lack of SA in Jordan supports the indicative nature of this research and opens the horizon to exploring new emotions toward shock adverts that may not have been reported earlier".

"Those adverts are more effective than what is used in Jordan, the ones used in Jordan are not shocking, and lack creativity. I am a cancer patient and an ex-smoker, I wish I were shocked when I was young and did not smoke ever" (M3-6:27 years, ex-smoker and a cancer survivor".

This research's participants stated that the "Current Anti-smoking Campaigns in Jordan are Useless". Yet, "the participants argued that SA must be creative, fresh, and evolving to have an impact on the target audience. However, there is a possibility that cigarette-sandwich advert will have an influence among Jordanians as most of the participants said communal views, and there was a general agreement that it took their attention with a very disgusting, offensive, and distasteful advert, which makes this advert effective, and shows the importance of creativity in advertising". A participant stated: "The cigarettes hamburger is disgusting, but it does not reflect reality. However, it is creative and unusual" (F3-1:24 year's old, smoker).

Most of the "participants showed acceptance of the idea of SA to be used in Jordan since it captured the viewer's attention because of the creativity and uncommon nature. Nonsmokers generally felt that they are victims of SHS".

"If a smoker views an image or television advert in which children are being harmed and are suffering from passive [second-hand] smoking, this smoker will start thinking about how he/she 
harms his/her children every time he/she smokes and will consider stopping smoking" (F6-4: 19 years old, nonsmoker).

\section{Shock advertising impact}

Three emerging subthemes explain the whole effect and power of SA: "Triggering Emotions, Memory Recall, and Concerns for SHS. The subtheme "Triggering Emotions" addresses the first objective in this research. Generally, participants had different emotions toward images reflecting different appeals and shock typology. The appeals applied in this research phase evoked negative emotions among all participants, smokers, and nonsmokers. While some images were neglected by participants, most of them were aroused by adverts and showed emotions that most likely will have a potential influence on smokers in the future".

Due to time constraints, "the two rounds of the focus groups were conducted four weeks apart. However, it enabled us to assess which adverts were most effective by exploring the participants' memory recall of adverts. An important indicator of the success of any advert is memory recall (Bushman, Phillips 2001). After the exposure, the adverts utilized for this research, $(n=36)$ of the participants exposed strong emotional reactions with repeated mentions to guilt, fear, sadness, and disgust. The remaining five participants did not mention any emotional reaction and felt uncertain about the adverts. It is vital to state that the researchers used various SA types as they explored a new culture since culture has a main effect on how consumers respond to SA" (Dahl, Frankenberger \& Manchanda 2003, Parry et al. 2013).

The cigarette sandwich advert raised emotions such as disgust among $(n=21)$ members of the focus groups "The most disgusting advert is the cigarette hamburger" (M6-1:26 years-old, nonsmoker). Even though it focuses on nonsmokers and contains a phrase that says, “Don't swallow other people's smoke" at the lower right side of the advert. "The participants perceived this advert as directed toward smokers, which shows that the strong creative advert dragged the participants' attention away from the information in the advert and they understood it in a different way than expected. This could be a main concern for marketers using SA: in for-profit marketing, the shock advert will also attract the attention of the audience and make them ignore the advertised brand; focus will be on the advert itself rather than the advertised brand" (Urwin, Venter 2014). 
Participants stated "fear" while viewing an advert that illustrates a lung cancer patient in the hospital. As a result, $(\mathrm{n}=19)$ "participants approved that it is shocking, horrendous, and sad. Emotions were like those evoked by the CONAC advert". However, participants responded to this advert differently than they did with the others probably, it addressed death "the image of the dead man on the cigarette pack in the Canada health advert is horrible. I do not smoke, but if I ever had the will to smoke and got exposed to this advert, I would choose not to smoke. I am still young; I do not want to die" (F3-3, a 20-year-old, nonsmoker).

In the second round of the focus groups, the research's "participants were asked to state their most unforgettable advert and the reasons behind recalling them. Amongst the nine adverts used in this research, the advert in an advertising campaign called 'Brown' illustrating a child in distress was the most remembered advert and still triggering emotions of sadness and guilt although they saw the advert four weeks ago. Therefore, the advert was remembered by 19 participants and when brought to discussion in the second round of the focus groups, many participants had emotional state toward it, and it was mentioned immediately. It has been clear that viewing shock adverts that aim to create awareness for SHS and there are children in the advert has a strong influence of emotions, in which the participants were able to remember this advert in specific".

A female participant mentioned that remembering the advert made her uncomforted " $I$ just felt uncomfortable while smoking next to my child" whereas a male participant expressed that: "memorizing the child made me feel sad". Furthermore, a male participant stated: "during this period, each time I smoke in the car while my child is next to me, after throwing the cigarette from the window I feel guilty. After viewing this image, I felt that I can't breathe! I can see the child in the advert every time I close my eyes or look at my children!"

Some participant indicated that they could not view the CONAC image again as it makes them very sad. A smoker male stated: "I don't think I can see this image again; it makes me sad and urges me to cry", likewise a female nonsmoker mentioned: "it makes me angry to imagine the harm that smokers cause to children, this seems a cause that we need to fight for. Ever since I saw the child's image, I have been thinking what could be done to save the children from smoker.s"

Stressing on "guilt for harming others and sadness due to the negative effects of smoking on children, from a participant's point of view, will be effective in Jordan and many similar countries". "It helps raise awareness among smokers, and helps them realize that they are hurting 
those innocent children by their behavior, as smoking next to others is a normal behavior for them. (M 6-5, 35 years old, smoker).

This research uncovers that including "adverts with children's pictures while blending them with emotional and fear appeals are effective for targeting Jordanian parents in specific, and Arabs in general. It was obvious that the shocking side in the child's advert triggered emotions of sadness, compassion, and empathy amongst participants, pointing out that such adverts are more likely to generate an awareness that might drive to change in SHS behaviors in Jordan."

"The child's picture is a good idea to show how adult smokers harm children if they smoke next to them" (M1-3, 24-year-old, smoker).

A significant sense that rises is that a certain "social marketing campaign may be unsuccessful in a certain culture even though it is successful in other cultures. Participants reported different attitudes toward quitting because of the cognitive differences among them (Veer et al., 2008). Regarding this, the Jordanian participants related differently with the adverts that depict innocent children more than they did with the advert of a cancer patient man". This can be useful for social marketers and policymakers in Jordan decipher what is truly shocking and effective to Jordanians. "It will influence smokers like me. When I saw the advert, I put myself in his situation. The Canada Health advert is effective and informative" (M 4-2: 25-year-old smoker).

\section{Drawbacks to shock advertising}

Participants "reported some limitations for shocking advertising that might restrict its effectiveness. These limitations were expressed in three subthemes: Accustomed to Shocking Images, Defensiveness triggered by Offensiveness, and Capturing but Ineffective”.

Some participants argued that "people in the Middle East have become "Accustomed to Shocking adverts" because of the daily over exposure in the media. Participants from two different groups stated that violence, aggression, fighting, and killing are widespread in video games, news, and movies, and thus, the image of a dead man or woman no longer causes shock". "Nowadays, death and blood images are in video games; in many games, there is killing, fighting, and blood, which isn't ethical......... think there are too many other problems in the world, and so using a child or a dead body is no longer effective" (F5-4: 19 years old, nonsmoker). 
Several participants stated that "smokers might be familiar to routine exposure to anti-smoking shocking adverts. Thus, there must be an extensive range of shocking adverts and they must be changed on a regular basis". One male smoker said,

"Repeated shock advertising means that people will get used to it, and in the long run, it will no longer have an effect. I might only be shocked once or twice, and so such adverts must be changed every now and again".

Several smoker participants "felt offended while arguing smoking as a risky consumption behavior, and their attitude was defensive and protective. This turned out to be obvious from their arguments about the anti-smoking advertising campaigns”. A 25-year-old female smoker (F1-2) said, "I think that people exaggerate. Smoking is not the only reason for cancer. Many people do not smoke, yet they have cancer". In this regard, "there could be several explanations behind this attitude. The nature of tobacco in all its forms is extremely addictive, leading some people to believe that any anti-smoking campaigns are ineffective, specifically it is accepted widely among the society".

"I don't need to be shocked or informed. I already know the negative effects of smoking on my health. Such adverts might be effective for few people but will not change the attitudes of all smokers. In my opinion, there are other ways to target smokers; smokers do not need to be shocked" (M2-5: 33 years, smoker).

In regard to the effectiveness of anti-smoking SA, 29 of the 41 participants showed a negative attitude, which turned out to be more obvious when participants stated that SA: "Capturing but Ineffectiv.e" However, "this attitude could be overcome by concentrating on creativity in antismoking SA campaigns, also by pursuing SHS rather than smoking in its firsthand influence as discussed earlier in this chapter. However, this number cannot be generalized as this is an inductive qualitative focus group based research and its outcomes call for and set a benchmark for further in-depth qualitative exploration and quantitative studies that could give more precise insights into this matter".

Most of the smoker participants "discussed that they did not need informative messages about the negative consequences of smoking on their health, as they were already aware of them. Though they approved that SA successfully captured their attention to a greater extent more than any other advertising strategy". A female participant recommended that "shock advertising can effectively get the point across. In a normal advert, we just look, read, and go, but shock advertising captures 
your attention". Most participants commented that the "effects of shock adverts would be very short term and would not likely change behaviors." 


\section{Discussion}

This research shows that "while the participants identified the adverts as shocking, in general, they were not affected by any of the images to the point of being motivated to quit smoking. This result contradicts with (Veer, Rank 2012) who stated that norm violation has a significant role in the effectiveness of advertising. Adverts must speak to the social norms of the societies they intend to target. Smokers in Europe are more aware that smoking is unfavorable, which reveals cultural variances between Arab societies and European societies with regards to this risky consumption behavior (Hamilton, Hassan 2010). European smokers are certain that other nonsmokers had negative views and attitudes regarding their risky consumption behavior, the time wasted on smoking, and the smell of smoke" (Hamilton, Hassan 2010).

It has been revealed in this research that "present anti-smoking campaigns depending on verbal messages and mild images had never been effective in targeting smoking in its various forms and effects. The use of more aggressive tactics could have a better effect on smokers, especially in their behaviors regarding SHS. The lack of shock adverts, along with the failure of current antismoking adverts could enhance the effect of anti-smoking campaigns using shocking images in the future, as the shocking component in anti-smoking advert will disrupt social norms (Manyiwa, Brennan 2012)in a region known for its conservative nature. Furthermore, evoking emotions, since shock adverts possibly will be stronger because of the reactive and sensitive nature of Arabic cultures (Hammad, Shah 2018) may lead to a behavioral modification that might be a result of strong emotions of guilt and regret" (Schulte 2007).

Emotions reported had a "positive influence in creating a potential behavioral change, specifically with regards to SHS. Guilt and fear have been connected to create significant behavioral change (Manyiwa, Brennan 2012). It is suggested that people develop a fear of the results of their actions leading them to feel guilty (Satas 2014). In the context of SA, guilt was mentioned as a vital motivator to make the target audience rethink their risky consumption behavior (Amodio, Devine \& Harmon-Jones 2007). Additionally, guilt has taken over from other emotions as a dominant human emotion and an effective motivator of change. Guilt is regarded a more beneficial emotion in influencing particular and corrective actions (Bedford et al. 2011). Employing guilt appeals in advertising is a common tactic used in social marketing (Lee-Wingate, Moon \& Bose 2014). Guilt appeals in social marketing most likely produce positive reactions toward the adverts and the cause of the advert" (Brennan, Binney 2010). 
Moreover, "fear is an effective persuader of behavioral change (Manyiwa, Brennan 2012). Parry et al., discuss that advertising is designed to invoke fear, offend and question cultural and social norms (Parry et al. 2013). In this research, this is essential as smoking seems a social norm in Jordan and targeting it with fear evocative adverts could lead to a behavioral change. Fear is connected to self-efficacy which could have an indirect and positive effect on attitudes toward the advert and the intention to change behavior" (Zlomuzica et al. 2015). Social marketing campaigns “mainly used for not-for-profit causes like anti-smoking advertising (Lennon, Rentfro \& O'Leary 2010) mostly awaken negative emotions (Taute, McQuitty \& Sautter 2011), and frighten consumers enough to foster behavioral and attitudinal changes (Parry et al. 2013). Fear in advertising is an effective prevention of harmful behaviors (Jones, Cunningham \& Gallagher 2010). Emotions of sadness, guilt, anger, and fear had their influence only on SHS behaviors rather than firsthand smoking behaviors. This outcome is supported by the findings reported in a research conducted in Massachusetts, USA where more than 1400 smokers were exposed to anti-smoking adverts for a period of two years and were followed up for related quitting in response to those adverts". In the latter research, "smokers were exposed to more than 200 anti-smoking adverts, however, the quitting rate was only increased by $10 \%$ when compared to the normal quitting rate amongst smokers who are not exposed to anti-smoking adverts (Durkin, Blades 2009). Along the same line, sadness was triggered because of adverts including children in distress. Sadness reflects a sense of compassion or empathy (Cockrill, Parsonage 2016) and, in this regard, could have a strong possibility to make behavioral changes amongst smokers regarding SHS affecting vulnerable individuals (Krstic 2007). In addition, evoking sadness and grief encourage the target audience to think about the consequences of the negative behavior they are engaging in. As a result, selecting an emotional strategy and its influence for social marketing campaigns should be stated clearly" (Chan, Chang 2013).

Moreover, the effect of "SA was gauged through testing memory recall of the adverts. While memory recall was explored after a four-week period from participants' exposure to SA in this research. Collectively, it was presumed that only shock adverts presenting children in distress had better memory recall and may have a positive lasting effect on smokers' everyday behaviors related to SHS. SA viewers will almost certainly recall the shocking pictures used, instead of the brand (Urwin, Venter 2014). This is consistent with our research findings, as most of the participants in this research remembered the child suffering from SHS, followed by the cigarette burger that is 
disgusting, creative, and unusual. Furthermore, in some adverts, the participants did not read the information in the advert and just remembered the child's picture. Several participants, in specific parents, recalled the child image. Also, they are more likely to mention that they may take into consideration quitting smoking (although this is a desire rather than an action)". Furthermore, SA is more likely to be recalled than other adverts (Halvadia, Patel \& PATEL 2011). Though, "the research results of (Urwin, Venter 2014) are consistent with the current research's findings, where not all shock adverts recall are related to its effectiveness. Shock advertising is more successful and useful when creativity or uniqueness is the main included element in the advert. Also, the research participants acknowledged that depicting children being harmed in advertising is the most effective because of the family-oriented values in the Jordanian culture and the larger Arab world, which aroused strong and intense emotions between the participants and deemed to have an emotional impact. Children are seen as innocent and in need of protection. Parents of both genders were affected dramatically by the images of children suffering because of SHS".

\section{Contribution}

\section{Theoretical contributions}

This research fills "the gap in the literature on SA and smoking by defining what shock adverts are the most effective, and which have the highest rates of consumer recall, in societies with a deeply entrenched culture of smoking. As inductive research, this research generated the following theory: "Shock advertising showing children in distress is effective in targeting smokers' behaviors related to SHS." This is considered advancement in research on SA, as previous studies were indecisive in assessing the extent to which SA could generate behavioral change" (Krstic 2007).

\section{Societal implications}

"Marketers, social marketers, and policymakers can take advantage of the outcomes of this research, as it emphasizes the success of SA in reshaping and modifying the behaviors amongst smokers, which possibly will help in setting strategies for targeting SHS in societies where smoking is prevalent, and anti-smoking regulations are not effective. Besides that, consumers are already aware of the negative consequences of smoking on their own health; social marketers and policymakers now have a main advertising strategy to at least avoid harming other people, in specific children with SHS, by designing shocking adverts that generate guilt, fear, and sadness, which could lead to the desired behavioral changes. Shocking images targeting SHS have been shown to be an effective tool in achieving this. Smokers usually respond to anti-smoking adverts 
with denial or defensiveness, but when it comes to causing harm to other individuals, they are not in a strong position to defend themselves or deny the harm they cause to others."

Anti-smoking adverts and campaigns "should shift their focus from firsthand smoking towards targeting SHS. This, proven effective in this research, could be a cost-effective solution. Many smoking cessation campaigns cost a lot, while the outcomes achieved were reported to be below expectations. Focusing on SHS would be effective, and if an anti-SHS culture could be embedded in the social norms, it could generate a huge amount of financial savings in the health expenditure. In the context of this research, anti-smoking campaigns should shift from the use of regular advertisings and verbal messages to SA. This shift could increase the campaigns more effectively".

\section{Limitations and future research directions}

The nature of the in-depth qualitative research of this research "granted and reinforced rich insights of how the research's participants responded in an emotional way regarding SA targeting smoking and SHS over a period of 4 weeks. However, though there are several benefits for conducting a qualitative research, some limitations should be addressed".

The findings of this research cannot be generalizable given the small sample size. However, "this research provided an insight of how Jordanians respond to SA, and we are expecting similar findings in other Arab countries given the similarity of cultural and religious beliefs between Jordan and may Arabic and Islamic countries. Furthermore, this research has been done in a collectivist culture country, Jordan, not an individualistic culture like Western Europe, as this factor has an impact on the effectiveness of SA" (Virvilaite, Matuleviciene 2013). 


\section{References}

Albert-Lőrincz, E., Szabó, B., Paulik, E. and Gáspárik, A.I. (2019), "Do Minorities Smoke More? A Comparison of Smoking Prevalence and Other Sociodemographic Factors Between Ethnic Romanian and Hungarian Teenagers in Romania", Journal of Romanian Literary Studies, , No. 16, pp. 112-119.

Albouy, J. and Décaudin, J. (2018), "Age differences in responsiveness to shocking prosocial campaigns", Journal of Consumer Marketing, Vol. 35, No. 3, pp. 328-339.

Amodio, D.M., Devine, P.G. and Harmon-Jones, E. (2007), "A dynamic model of guilt: Implications for motivation and self-regulation in the context of prejudice", Psychological Science, Vol. 18, No. 6, pp. 524-530.

Bedford, T., Collingwood, P., Darnton, A., Evans, D., Getersleben, B., Abrahamse, W. and Jackson, T. (2011), "Guilt: an effective motivator for pro-environmental behaviour change?", RESOLVE Working Paper Series, 07-11, .

Brainfluence, D.R. (2012), 100 ways to persuade and convince consumers with neuromarketing translated by Anonymous John Wiley \& Sons, Hoboken, NJ, USA.

Brennan, L. and Binney, W. (2010), "Fear, guilt, and shame appeals in social marketing", Journal of business Research, Vol. 63, No. 2, pp. 140-146.

Burki, T.K. (2019), "Tobacco control in Jordan", The Lancet respiratory medicine, Vol. 7, No. 5, pp. 386.

Bushman, B.J. and Phillips, C.M. (2001), "If the television program bleeds, memory for the advertisement recedes", Current directions in psychological Science, Vol. 10, No. 2, pp. 4347.

Chan, K. and Chang, H. (2013), "Advertising to Chinese youth: A study of public service ads in Hong Kong", Qualitative Market Research: An International Journal, Vol. 16, No. 4, pp. 421-435.

Charmaz, K. and Thornberg, R. (2020), "The pursuit of quality in grounded theory", Qualitative Research in Psychology, , No. DOI:10.1080/14780887.2020.1780357.

Clarke, V. and Braun, V. (2014), "Thematic analysis", in T. Teo(Ed.), Encyclopedia of critical psychology Springer, New York, pp. 1947-1952.

Cleland, J. (2015), "Exploring versus measuring: considering the fundamental differences between qualitative and quantitative research", Researching medical education, , pp. 1-14.

Cockrill, A. and Parsonage, I. (2016), "Shocking people into action: Does it still work?: An empirical analysis of emotional appeals in charity advertising", Journal of Advertising Research, Vol. 56, No. 4, pp. 401-413. 
Corbin, J.M. and Strauss, A. (1990), "Grounded theory research: Procedures, canons, and evaluative criteria", Qualitative sociology, Vol. 13, No. 1, pp. 3-21.

Dahl, D.W., Frankenberger, K.D. and Manchanda, R.V. (2003), "Does it pay to shock? Reactions to shocking and nonshocking advertising content among university students", Journal of Advertising Research, Vol. 43, No. 3, pp. 268-280.

Dann, S. (2010), "Redefining social marketing with contemporary commercial marketing definitions", Journal of Business research, Vol. 63, No. 2, pp. 147-153.

Darawad, M.W., Rezk-Hanna, M., Alhussami, M., Lee, J., Mostafa, A., Abu-Rmeileh, N., Kheirallah, K., Salama, M., Nakkash, R. and Saleh, Z. (2020), "Poly-Tobacco Use among Young Adult Waterpipe Smokers: Insights from University Students in Three Eastern Mediterranean Countries", Substance use \& misuse, Vol. 55, No. 13, pp. 2099-2108.

Durkin, K. and Blades, M. (2009), "Young people and the media: Special issue introduction", British Journal of Developmental Psychology, Vol. 27, No. 1, pp. 1-12.

Ekpu, V.U. and Brown, A.K. (2015), "The economic impact of smoking and of reducing smoking prevalence: review of evidence", Tobacco use insights, Vol. 8, pp. TUI. S15628.

Engelbart, S.M., Jackson, D.A. and Smith, S.M. (2017), "Examining Asian and European reactions within shock advertising", Asian Journal of Business Research, Vol. 7, No. 2, pp. $37-56$.

Farrelly, M.C., Duke, J.C., Davis, K.C., Nonnemaker, J.M., Kamyab, K., Willett, J.G. and Juster, H.R. (2012), "Promotion of smoking cessation with emotional and/or graphic antismoking advertising", American Journal of Preventive Medicine, Vol. 43, No. 5, pp. 475-482.

Feigl, A.B., Salomon, J.A., Danaei, G., Ding, E.L. and Calvo, E. (2015), "Teenage smoking behaviour following a high-school smoking ban in Chile: interrupted time-series analysis", Bulletin of the World Health Organization, Vol. 93, pp. 468-475.

Gooch, K. and Sheldon, D. (2019), "Holiday camps, prison time and confined escapism: Understanding leisure, pleasure and harm in prisons", in T. Raymen \& O. Smith(Eds.), Deviant Leisure Palgrave Macmillan, Cham, pp. 403-423.

Haddad, L.G., Al-Zyoud, S., Baker, N.A., Gharaibeh, H., Shahawy, O.E. and Alramadhani, R. (2011), "Secondhand smoking in Jordan: Clearing the air for one of the highest tobacco prevalence countries in the Middle East", Tobacco Use Insights, Vol. 4, No. 1, pp. 1-7.

Halvadia, N., Patel, R. and PATEL, S. (2011), "Shock Advertising and its impact", International Journal of Sales and Marketing Management, Vol. 1, No. 1, pp. 30-36.

Hamilton, K. and Hassan, L. (2010), "Self-concept, emotions and consumer coping: Smoking across Europe", European Journal of Marketing, Vol. 44, No. 7/8, pp. 1101-1120. 
Hammad, W. and Shah, S. (2018), "Dissonance Between the "International" and the Conservative "National": challenges facing school leaders in international schools in Saudi Arabia", Educational Administration Quarterly, Vol. 54, No. 5, pp. 747-780.

Hammal, F., Mock, J., Ward, K.D., Eissenberg, T. and Maziak, W. (2008), "A pleasure among friends: how narghile (waterpipe) smoking differs from cigarette smoking in Syria", Tobacco control, Vol. 17, No. 2, pp. e3.

Hastings, G. (2007), Social marketing: Why should the devil have all the best tunes? translated by Anonymous Butterworth-Heinemann, Burlington, MA.

Huque, R., Dogar, O., Cameron, I., Thomson, H., Amos, A. and Siddiqi, K. (2015), "Children learning about second-hand smoking: A feasibility cluster randomized controlled trial", Nicotine \& Tobacco Research, Vol. 17, No. 12, pp. 1465-1472.

Jabbar, F.A. and Christopher, A.A. (2012), "Negative emotional appeals in online attorneys' marketing in North America", International Journal of Social Science and Humanity, Vol. 2, No. 4, pp. 305.

Jin, H.S., Kerr, G. and Suh, J. (2019), "Impairment effects of creative ads on brand recall for other ads", European Journal of Marketing, Vol. 53, No. 7, pp. 1466-1483.

Jones, T., Cunningham, P.H. and Gallagher, K. (2010), "Violence in advertising", Journal of Advertising, Vol. 39, No. 4, pp. 11-36.

Kamal, M. (2017), A Survey on Smoking Status and Knowledge regarding the Consequences of Smoking among the people of Dhaka City, East West University.

Karasneh, R., Al-Azzam, S., Nusair, M. and Hawamdeh, S. (2021), "Perceptions, symptoms, and practices of electronic cigarette users: Descriptive analysis and validation of Arabic short form vaping consequences questionnaire", Plos one, Vol. 16, No. 1, pp. e0245443.

Keller, C., Vega-López, S., Ainsworth, B., Nagle-Williams, A., Records, K., Permana, P. and Coonrod, D. (2014), "Social marketing: approach to cultural and contextual relevance in a community-based physical activity intervention", Health promotion international, Vol. 29, No. 1, pp. 130-140.

Knishkowy, B. and Amitai, Y. (2005), "Water-pipe (narghile) smoking: an emerging health risk behavior", Pediatrics, Vol. 116, No. 1, pp. e113-9.

Krstic, T. (2007), Attitudes toward shock advertising of Western-European and Serbian university students with regard to public health context (anti-smoking and anti-HIV/AIDS campaigns), University of Nottingham.

Kusel, J., Timm, B. and Lockhart, I. (2013), "The impact of smoking in the home on the health outcomes of non-smoker occupants in the UK", Tobacco induced diseases, Vol. 11, No. 1, pp. 1-10. 
Lee, N. and Kotler, P. (2011), Social marketing: Influencing behaviors for good, 4th ed., translated by Anonymous Sage, Thousand Oaks, CA.

Lee-Wingate, S.N., Moon, J.Y. and Bose, M. (2014), "The influence of mortality focus on guilt advertising effectiveness", Journal of Marketing Theory and Practice, Vol. 22, No. 1, pp. 103-114.

Lennon, R., Rentfro, R. and O'Leary, B. (2010), "Social Marketing and Distracted Driving Behaviors among Young Adults: The Effectiveness of Fear Appeals", Academy of Marketing Studies Journal, Vol. 14, No. 2, pp. 95.

Lincoln, Y. and Guba, E. (1985), Naturalistic Inquiry translated by Anonymous Sage Publications, Newbury Park, CA.

Machová, R., Huszárik, E.S. and Tóth, Z. (2016), "Is Advertising Innovation the Same as Shocking?", Editorial Board, Vol. 11, No. 2, pp. 40.

Maguire, M. and Delahunt, B. (2017), "Doing a thematic analysis: A practical, step-by-step guide for learning and teaching scholars.", All Ireland Journal of Higher Education, Vol. 9, No. 3.

Manyiwa, S. and Brennan, R. (2012), "Fear appeals in anti-smoking advertising: How important is self-efficacy?", Journal of Marketing Management, Vol. 28, No. 11-12, pp. 1419-1437.

Nan, X., Zhao, X., Yang, B. and Iles, I. (2015), "Effectiveness of cigarette warning labels: examining the impact of graphics, message framing, and temporal framing", Health communication, Vol. 30, No. 1, pp. 81-89.

Parry, S., Jones, R., Stern, P. and Robinson, M. (2013), "'Shockvertising': An exploratory investigation into attitudinal variations and emotional reactions to shock advertising", Journal of Consumer Behaviour, Vol. 12, No. 2, pp. 112-121.

Prendergast, G., Ho, B. and Phau, I. (2002), "A Hong Kong view of offensive advertising", Journal of marketing communications, Vol. 8, No. 3, pp. 165-177.

Rice, R. and Hancock, L. (2005), "The mall intercept: A social norms marketing research tool", The Report on Social Norms, Vol. 4, No. 7, pp. 4-7.

Safi, M. and Al-Tahat, J. (2020), , Jordan smoking rates highest in world amid claims of big tobacco interference [Homepage of The Guardian], [Online]. Available:

https://www.theguardian.com/world/2020/jun/23/jordan-smoking-rates-highest-in-worldamid-claims-of-big-tobacco-interference [2021, Apr/09].

Satas, D. (2014), Exploring the impact of shock advertisement on students with culture as influential factor, Dublin Business School.

Schiffman, L.G. and Wisenblit, J.L. (2019), Consumer Behavior, 12th ed., translated by Anonymous Pearson, England. 
Schulte, S.K. (2007), "Avoiding Culture Shock: Using Behavior Change Theory to Implement Quality Improvement Programs", Journal of AHIMA, Vol. 78, No. 4, pp. 52-56.

Shishani, K., Nawafleh, H., Jarrah, S. and Froelicher, E.S. (2011), "Smoking patterns among Jordanian health professionals: a study about the impediments to tobacco control in Jordan", European Journal of Cardiovascular Nursing, Vol. 10, No. 4, pp. 221-227.

Sibai, A.M., Iskandarani, M., Darzi, A., Nakkash, R., Saleh, S., Fares, S. and Hwalla, N. (2016), "Cigarette smoking in a Middle Eastern country and its association with hospitalisation use: a nationwide cross-sectional study", BMJ open, Vol. 6, No. 4, pp. e009881.

Simpson, J.K. (2017), "Appeal to fear in health care: appropriate or inappropriate?", Chiropractic \& manual therapies, Vol. 25, No. 1, pp. 1-10.

Taute, H.A., McQuitty, S. and Sautter, E.P. (2011), "Emotional information management and responses to emotional appeals", Journal of Advertising, Vol. 40, No. 3, pp. 31-44.

Urwin, B. and Venter, M. (2014), "Shock advertising: Not so shocking anymore. An investigation among Generation Y", Mediterranean Journal of Social Sciences, Vol. 5, No. 21, pp. 203.

Veer, E. and Rank, T. (2012), "Warning! The following packet contains shocking images: the impact of mortality salience on the effectiveness of graphic cigarette warning labels", Journal of Consumer Behaviour, Vol. 11, No. 3, pp. 225-233.

Virvilaite, R. and Matuleviciene, M. (2013), "The impact of shocking advertising to consumer buying behavior", Economics and management, Vol. 18, No. 1, pp. 134-141.

World Health Organization (2020), , Tobacco Fact Sheet. Available: https://www.who.int/newsroom/fact-sheets/detail/tobacco [2021, April/9].

Wright, E., Khanfar, N.M., Harrington, C. and Kizer, L.E. (2016), "The lasting effects of social media trends on advertising", Journal of Business \& Economics Research (JBER), Vol. 14, No. 3, pp. 75-82.

Zlomuzica, A., Preusser, F., Schneider, S. and Margraf, J. (2015), "Increased perceived selfefficacy facilitates the extinction of fear in healthy participants", Frontiers in behavioral neuroscience, Vol. 9, pp. 270.

Acknowledgment: The first author is grateful to the Applied Science Private University, Amman, Jordan for the financial support granted to this research project. 


\section{Appendix one: Focus Groups interview guide}

\section{Focus group I}

Participants were exposed to a set of advertisements and asked the following questions

1. "What is your general opinion about this type of marketing communication strategies"?

2. "Do you perceive shock advertisements as creative, offending, or both? Could you give a reason to your answer"?

3. "Do you think this type of advertisements is shocking? Could you give a reason to your answer"?

4. "What would you find shocking in an advertisement"?

5. "Which advertisements did you find most striking"?

6. "Could you give a reason to your answer"?

7. "Can you please describe your feelings? Which advertisements do you still remember? Could you give a reason to your answer"?

8. "Do you find using shock advertising ethically justified"?

9. "Which advertisement can have an influence on your attitude and behavioral intention? And why"?

\section{Part 2: Focus Group II Questions (four weeks after the first session)}

1. "What advertisements do you remember from our last meeting and why? What feelings does this advertisement bring to you"?

2. "Do you think that shock advertising is useful in creating social and health awareness? And why"?

3. "Should Shock advertising be used for creating social awareness? If so, how"?

4. "Would you find using shock advertising for non-profit purposes is justified"?

5. "Do you think that shock advertising is effective as a marketing communication strategy in the social marketing context? Can you please explain why you think that? And can you give me an example"?

6. "Regarding non-profit advertising, which of the following would be the most appealing, shock, humour, information, fear, warmth, use of celebrities, emotional"?

7. "Where and how do you think those kinds of advertisements will benefit the society in terms of anti-smoking advertisements and cancer awareness advertisements"?

8. "What is the best way to get a social marketing message related to health awareness? (Online, billboard, magazines, newspapers, TV, etc...)".

9. In your opinion, "what are the most effective marketing strategies that can be used and applied to create social and health awareness in Jordan? Emotion, fear, information, humour, etc..."? 